\title{
Tendencia temporal y evaluación demográfica de hospitalizaciones por osteoartritis
}

\section{Temporal Trends and Demographic Evaluation of Hospitalizations Due to Osteoarthritis}

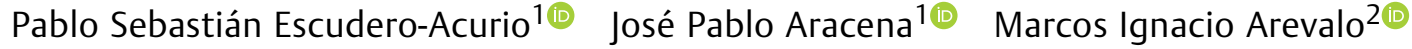 \\ Carlos Alonso Escudero 2,3 (1)
}

1 Escuela de Medicina, Facultad de Medicina y Ciencia, Universidad San Sebastián, Concepción, Chile

2 Laboratorio de Fisiología Vascular, Departamento de Ciencias Básicas, Universidad del Bío-Bío, Chillán, Chile

${ }^{3}$ Grupo de Investigación e Innovación en Salud Vascular (GRIVAS Health), Chillán, Chile
Address for correspondence Carlos Escudero, MD, PhD, Vascular Physiology Laboratory, Group of Research and Innovation in Vascular Health (GRIVAS Health), Basic Sciences Department, Faculty of Sciences, Universidad del Bio-Bio, Fernando May Campus, Chillán, Chile, Andres Bello Av, 720, Chillán, Chile (e-mail: cescudero@ubiobio.cl).

Rev Chil Ortop Traumatol 2021;62(2):e84-e92.

\section{Resumen \\ Palabras Clave \\ - osteoartritis \\ - prevalencia \\ - egresos hospitalarios \\ - Chile \\ - artrosis de cadera \\ - artrosis de rodilla \\ Objetivo Describir la tendencia temporal de osteoartritis (OA) según egresos hospitalarios considerando ubicación anatómica, distribución por sexo, edad, condiciones más frecuentes, y ubicación geográfica en Chile entre los años 2012 y 2018. \\ Metodología Estudio descriptivo, retrospectivo, poblacional, que analizó las bases de datos públicas del Departamento de Estadísticas e Información en Salud (DEIS) del Ministerio de Salud de Chile, en particular los registros con codificación de M15 a M19. Se calcularon las tasas por 100 mil egresos hospitalarios, así como también el porcentaje de OA según las categorías analizadas. \\ Resultados Se encontraron 11.622.605 egresos hospitalarios entre 2012 y 2018 . El número total de egresos hospitalarios por OA fue de 78.700. Se observó que la distribución por sexo fue similar a lo largo de los años ( 40\% y $60 \%$ en hombres y mujeres, respectivamente). El rango de edad más afectado fue el de 65 a 79 años, cuya tasa promedio fue de 2.046 por 100 mil egresos; además, fue el grupo que presentó el mayor aumento (60\%) en su tasa de 2012 a 2018. La Región Metropolitana (291,7 egresos) y de Valparaíso $(89,6$ egresos) presentaron las tasas más altas. La cadera $(56,6 \%)$ y la rodilla (31\%) fueron las ubicaciones más frecuentes de casos de OA, con distribución similar por sexo, edad y geografía en comparación a los egresos totales por OA. \\ Conclusiones Los egresos hospitalarios por OA en Chile aumentaron entre 2012 y 2018, preferencialmente en casos de OA en las articulaciones de cadera y rodilla en el grupo de mujeres de edad avanzada de la zona centro del país. \\ Nivel de evidencia: Estudio descriptivo}

recibido

21 de agosto de 2020

aceptado

04 de febrero de 2021
DOI https://doi.org/

10.1055/s-0041-1735549. ISSN $0716-4548$.

\author{
(c) 2021. Sociedad Chilena de Ortopedia y Traumatologia. All rights \\ reserved. \\ This is an open access article published by Thieme under the terms of the \\ Creative Commons Attribution-NonDerivative-NonCommercial-License, \\ permitting copying and reproduction so long as the original work is given \\ appropriate credit. Contents may not be used for commercial purposes, or \\ adapted, remixed, transformed or built upon. (https://creativecommons.org/ \\ licenses/by-nc-nd/4.0/) \\ Thieme Revinter Publicações Ltda., Rua do Matoso 170, Rio de \\ Janeiro, RJ, CEP 20270-135, Brazil
}


Abstract

\section{Keywords}

- osteoarthritis

- prevalence

- hospital discharges

- Chile

- hip osteoarthritis

- knee osteoarthritis
Objective To describe the temporal trends of osteoathritis (OA) according to hospital discharges considering anatomical location, distribution by gender, age, more frequent conditions, and geographic location in Chile between 2012 and 2018.

Methodology A retrospective, descriptive and population study which analyzed the public databases of the Department of Health Statistics and Information (Departamento de Estadísticas e Información en Salud, DEIS, in Spanish) of the Ministry of Health of Chile, in particular the records with codes M15 through M19. Rates per 100 thousand hospital discharges were calculated, as well as the percentage of OA according to the categories analyzed.

Results A total of 11,622,605 hospital discharges were found between 2012 and 2018. The total number of hospital discharges due to OA was of 78,700 . The distribution by gender was similar over the years ( $\sim 40 \%$ and $60 \%$ among men and women respectively). Adults aged between 65 and 79 years were the most affected, and their average rate was of 2,046 per 100 thousand discharges. It was also the group that presented the highest increase (60\%) in rate from 2012 to 2018 . The Metropolitan Region (291.7 discharges) and Valparaíso (89.6 discharges) presented the highest rates in Chile. The hip (56.6\%) and knee (31\%) were the most frequent locations of OA, which presented similar distribution by gender, age and geography when compared with the total discharges due to OA.

Conclusions Hospital discharges due to OA in Chile increased between 2012 and 2018, preferentially in cases of hip and knee-joint OA in the group of elderly women in the central area of the country.

Level of evidence: Descriptive study

\section{Introducción}

La osteoartritis (OA) o artrosis se caracteriza por pérdida progresiva del cartílago articular, hipertrofia ósea marginal (osteofitos), y cambios en la membrana sinovial. ${ }^{1,2}$ La etiología de la OA no es totalmente entendida, ${ }^{2}$ aunque se conoce una serie de factores que participan en la patogenia, tanto biomecánicos (sobrecarga o esfuerzo físico) como la liberación de mediadores proinflamatorios (interleuquina 1 [IL-1]; factor de necrosis tumoral alfa [TNF- $\alpha$ ]) y actividad de proteasas (enzimas que degradan proteínas) intraarticulares. ${ }^{3}$ Todos estos factores inducen un estado inflamatorio del cartílago articular, el cual, manifestado crónicamente, se convierte en el componente fundamental de la fisiopatología de la OA. ${ }^{2-4}$

Existen diferentes maneras de clasificar la OA: por criterios radiológicos, clínicos o anatómicos. ${ }^{4}$ Por otro lado, también se clasifican según las articulaciones afectadas. Así, la OA puede afectar articulaciones "típicas" (cadera, rodilla, mano), las que habitualmente corresponden a formas primarias (relacionadas con edad). Por otro lado, puede afectar articulaciones "atípicas" (metacarpofalángica, codo, tobillo), que habitualmente corresponden a formas secundarias (relacionadas con otras patologías). ${ }^{5}$ Entre las principales manifestaciones clínicas de la OA se encuentra el dolor, rigidez y limitación del rango de movimiento de las articulaciones afectadas, ${ }^{6}$ mientras que, con la progresión de la enfermedad, se pueden apreciar deformaciones y cambios macroestructurales. ${ }^{7}$ Además, la OA se asocia a una importante morbilidad, que incluye la incapacidad funcional, siendo la causa más común de incapacidad permanente a nivel mundial, ${ }^{8}$ lo cual, a su vez, impacta negativamente en la calidad de vida de los individuos afectados. $^{9}$

La distribución etaria de esta enfermedad indica que el grupo de más baja prevalencia son los individuos menores de 40 años, ${ }^{10}$ siendo infrecuente en individuos menores de 20 años. Por otro lado, esta patología aumenta entre los individuos de 40 y 60 años, alcanzando la mayor tasa en las edades más avanzadas ( $>60$ años). ${ }^{11}$ Además, se presenta preponderantemente en mujeres. ${ }^{7,9}$ De hecho, se ha estimado que $18 \%$ de las mujeres de 60 años tienen OA sintomática, comparado con $9.6 \%$ de los hombres de la misma edad. ${ }^{12}$ La incidencia de las OAs de cadera y rodilla son las más frecuentes, en particular en países desarrollados. ${ }^{9}$

Sólo en Estados Unidos se ha estimado que cerca de 27 millones de personas fueron afectadas por OA en $2014 .^{4}$ Por otro lado, la prevalencia de OA de rodilla diagnosticada según criterios radiográficos o clínicos fue de $25.4 \%$ o $15.4 \%$ respectivamente, en un estudio en Suecia publicado en $1970 .^{13}$ En Latinoamérica, según el Programa Orientado a la Comunidad para el Control de Enfermedades Reumáticas (Community Oriented Program for the Control of Rheumatic Diseases, COPCORD, en inglés), la prevalencia de OA fue de $10.5 \%$ en México en $2011,{ }^{14}$ de $4.1 \%$ en Brasil en $2004,{ }^{15}$ y de $14.4 \%$ en Perú, en 2009, en individuos mayores de 35 años. ${ }^{16}$ 
En 2019 en Chile, la OA dio cuenta del 16.1\% de las causas de dolor crónico, encontrándose en segundo lugar por debajo del lumbago (22.1\%). ${ }^{17}$ A pesar de estos números, estudios epidemiológicos detallados y por regiones son escasos en Latinoamérica.

En Chile, la implementación del conjunto de beneficios Garantías Explícitas en Salud (GES) busca garantizar el acceso a la atención de salud a personas con ciertas patologías que han sido elegidas por su alto impacto sanitario y social. En estas garantias está incluido el tratamiento médico de las OAs de cadera y rodilla leves a moderadas en personas mayores de 55 años, pero sólo el tratamiento quirúrgico de la OA de cadera con limitación funcional severa en los mayores de 65 años (Decreto 170, Ministerio de Salud, 2005). ${ }^{18}$ Además, contamos con guías clínicas oficiales emitidas por el Ministerio de Salud, ${ }^{19}$ lo cual hace recomendaciones sobre el manejo de las OAs. Pese a ello, no existe una actualización reciente que incluya, por ejemplo, datos epidemiológicos que evidencien la magnitud del problema de la OA en la salud pública de nuestro país. Sin embargo, el Departamento de Estadísticas e Información en Salud (DEIS) del Ministeria de Salud de Chile reporta los egresos hospitalarios en forma anual a nivel nacional, y desglosado por región y sexo. Estos reportes permiten evaluar la evolución de los egresos hospitalarios con diagnóstico de OA, que, en su mayoría, recibieron tratamiento quirúrgico de su patología, incluyendo aquellos pacientes con OA de cadera de resolución quirúrgica cubiertos por el GES; o con OA de rodilla, que, aunque no están cubiertos por el GES, recibieron también tratamiento quirúrgico.

Por tanto, el objetivo del presente trabajo es describir la tendencia temporal, y la distribución según sexo, edad, y localización geográfica de las hospitalizaciones por OA en Chile entre 2012 y 2018. Además, enfatizamos la descripción de los egresos hospitalarios más frecuentes según la ubicación anatómica de la OA.

\section{Materiales y Métodos}

Este es un estudio retrospectivo, descriptivo, poblacional de los egresos hospitalarios de OA ocurridos entre 2012 y 2018 en Chile. Los registros fueron descargados de las bases de datos públicas del DEIS.

Para la búsqueda, se seleccionaron las bases de datos correspondientes a los años analizados según causa y edad. En dichas bases, las patologías están codificadas según la décima revisión de la Clasificación Internacional de Enfermedades (CIE10). Se utilizaron los registros con codificación de M15 a M19, que corresponden a la patología de $\mathrm{OA}$, incluyendo su clasificación. Puntualmente, se identifican: poliartrosis (M15); coxartrosis (M16); gonartrosis (M17); OA de la primera articulación carpo-metacarpiana (M18) y otras OAs (M19).

Se calcularon las tasas por 100 mil egresos hospitalarios. Para ello, se utilizó la fórmula:

$$
\text { Tasa de egresos por } X=\frac{\text { Número de casos } * 100.000}{\text { Egresos totales por año }}
$$

en que X significa la categoría (sexo, edad, ubicación) a analizar.
Se presentan las tasas de egreso hospitalario según sexo, edad, clasificación anatómica, y ubicación geográfica, así como también el porcentaje según las categorías analizadas. Finalmente, se enfatiza la descripción de las dos OAs más frecuentes según la ubicación anatómica.

Para el análisis, se fabricó una base de datos secundaria para facilitar los cálculos de las tasas y porcentajes en el programa Microsoft Excel (Microsoft Corp., Redmond, WA, EEUU). Adicionalmente, los gráficos han sido hechos en los programas de GraphPad Prism (GraphPad Software, Inc., San Diego, CA, EEUU), versión 8, e Infogram (Prezi, San Francisco, CA, EEUU).

\section{Resultados}

Durante el periodo analizado, se encontraron en total 11.622.605 egresos hospitalarios en el país, con ligeras variaciones anuales, que van desde los 1.670 .447 egresos en 2012 a 1.669 .602 egresos totales en 2018 (-Tabla 1).

Las tasas de egreso hospitalario por OA muestran una clara tendencia al alza: de 556,5 en 2012 a 896,2 por $100 \mathrm{mil}$ egresos totales en 2018. En el periodo analizado, hubo un total 78.700 hospitalizaciones por OA, y se observó que la distribución por sexo fue similar a lo largo de los años, manteniéndose en promedio una representación del $40 \%$ en los hombres y del 60\% en las mujeres ( - Tabla 1), lo que se tradujo en 31.688 hombres y 47.011 mujeres hospitalizados por OA.

Al analizar los egresos según distribución por edad, observamos que las tasas tienden al alza en todos los grupos etarios (-Tabla 2). En específico, la OA es más frecuente entre los rangos de edad que van desde 45 a más de 80 años, quienes muestran una tasa promedio de 3.994 por 100 mil egresos hospitalarios. De este grupo, sobresalen aún más aquellos en el rango de edad entre los 65 y 79 años, cuya tasa promedio fue de 2.046 por 100 mil egresos, y que es el grupo que presenta el mayor aumento, con 60\% de crecimiento en su tasa entre 2012 y 2018, con un registro de más de 6.800 egresos hospitalarios en 2018 (-Tabla 2).

Con respecto a la distribución en las regiones de Chile, la zona centro concentra la mayor cantidad. En contraste, los extremos del país, tanto la zona norte como austral, muestran la menor tasa (-Figura 1A). Las regiones que presentaron en promedio las tasas más altas fueron la Región Metropolitana (291,7 egresos) y la Región de Valparaíso (89,6 egresos); en cuanto a las tasas más bajas, fueron la Región de Aysén (4,2 egresos) y la Región de Arica y Parinacota (7,6 egresos).

Cuando se analizó de forma dirigida la diferencia en las tasas para evaluar la magnitud de aumento, encontramos que los egresos hospitalarios aumentaron en la mayoría de las regiones de Chile. La mayor diferencia se encontró en las Regiones Metropolitana y Bío-bío (-Figura 1B). Además, la tasa por 100 mil egresos en la Región de la Araucanía aumentó 5,5 veces en el periodo analizado, seguida por la Región del Maule (2,5 veces).

En cuanto a la clasificación topográfica de la OA, pudimos observar poca variabilidad considerando el total de egresos 


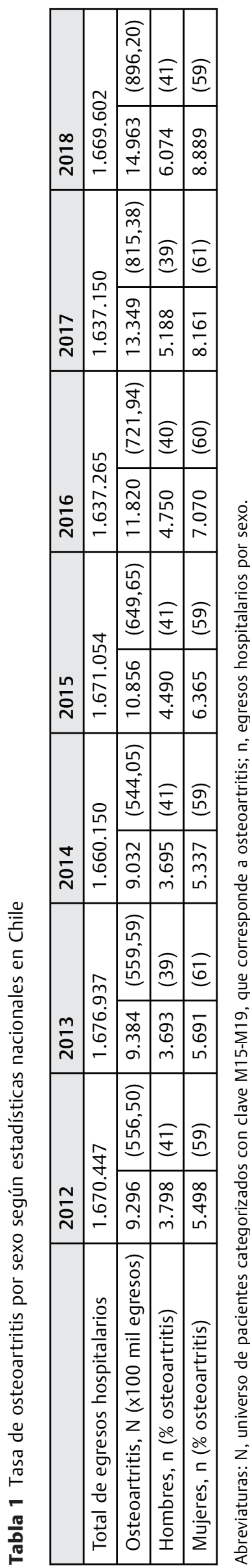

(-Tabla 3). Los egresos por coxartrosis (OA de cadera) y gonartrosis (OA de rodilla) corresponden en promedio a $56,6 \%$ y $31 \%$, respectivamente, del total registrado por el DEIS con diagnóstico de OA. Por ello, describimos la situación de estas localizaciones de OA en relación a su evolución en el tiempo, sexo, edad, y la distribución geográfica.

A lo largo del periodo analizado, se registraron más de 44 mil hospitalizaciones por coxartrosis en el país, con un aumento temporal de $80 \%$. La tasa en 2018 fue de 493 por 100 mil egresos totales ( $\mathbf{- F i g u r a ~ 2 A )}$. En forma similar a los egresos por $\mathrm{OA}$, se observó que la tasa de egresos por coxartrosis fue más alta en mujeres (62\%) que en hombres (38\%), con un registro de más de 27 mil egresos de mujeres afectadas (-Figura 2B).

Observamos aumentos en la tasa de egresos de coxartrosis en todas las edades: un aumento promedio de $29 \%$ de 2012 a 2015 , y de $27 \%$ de 2015 a 2018. Además, entre 2012 y 2018, en los menores de 20 años, se duplicó la tasa de egresos, mientras que, en el rango etario entre 65 y 79 años, ésta aumentó en $59 \%$ (- Figura 2C).

Por otro lado, en el mismo periodo, la gonartrosis fue registrada en 24.728 egresos hospitalarios, y el análisis temporal indica un aumento de más del doble, llegando a una tasa de 316 por 100 mil egresos en 2018 ( - Figura 3A). Su comportamiento en cuanto a sexo es similar a la coxartrosis, en que las tasas de egreso en el sexo femenino son las más altas ( - Figura 3B). En cuanto a la edad, el rango etario de 65 a 79 años mostró la mayor tasa: más de 5.600 egresos en los años 2012, 2015 y 2018 (-Figura 3C).

A nivel de regiones de Chile, se observó que la gonartrosis y coxartrosis tenían comportamientos similares, ya que en ambas ocurrió un aumento de las tasas en casi todas las regiones ( - Figura 4A y 4B). El mayor número se encuentra en la Región Metropolitana: más de 2.200 egresos por coxartrosis y más de 3.400 por gonartrosis. Además, en números brutos, las regiones Metropolitana, del Bío-bío, y del Maule presentaron la mayor diferencia en las tasas (-Figura 4). Finalmente, analizamos la magnitud del aumento por región; la que ha experimentado el mayor aumento fue la Araucanía: de 7,2 veces para egresos por coxartrosis. Para la gonartrosis, las regiones del Maule $(5,2)$ y la Araucanía $(4,7)$ experimentaron el mayor aumento en los egresos hospitalarios por gonartrosis.

\section{Discusión}

Los egresos hospitalarios por OA fueron de 78.700 en todo Chile entre 2012 y 2018. En este estudio, mostramos la evolución de esta condición según los datos oficiales del Ministerio de Salud: hubo una tendencia mantenida al alza principalmente en la zona centro-sur de Chile, que afectó mayoritariamente a mujeres y a la población adulta. Las condiciones más frecuentes fueron la coxartrosis y la gonartrosis.

Estudios similares en Suecia ${ }^{20}$ y en el Reino Unido ${ }^{21}$ determinaron un aumento general de las hospitalizaciones por OA, y una tendencia al alza entre 1998 y 2018. La OA afecta principalmente a mujeres, y las causas de esta mayor 
Tabla 2 Tasa de osteoartritis por edad según estadísticas nacionales en Chile

\begin{tabular}{|l|l|l|l|l|l|l|l|}
\hline Edad (años) & 2012 & 2013 & 2014 & 2015 & 2016 & 2017 & 2018 \\
\hline$<20$ & 24,6 & 28,7 & 31,7 & 35,3 & 40,0 & 26,8 & 31,3 \\
\hline $20-44$ & 172,7 & 156,7 & 157,7 & 176,2 & 202,3 & 184,6 & 204,5 \\
\hline $45-64$ & $1.063,5$ & 987,3 & 975,2 & $1.080,0$ & $1.218,0$ & $1.375,5$ & $1.544,3$ \\
\hline $65-79$ & $1.658,7$ & $1.729,3$ & $1.644,7$ & $2.059,9$ & $2.131,8$ & $2.451,3$ & $2.648,8$ \\
\hline$>80$ & 648,8 & 716,2 & 692,3 & 763,3 & 825,2 & 887,1 & 863,3 \\
\hline
\end{tabular}

Nota: Tasa por 100 mil egresos.
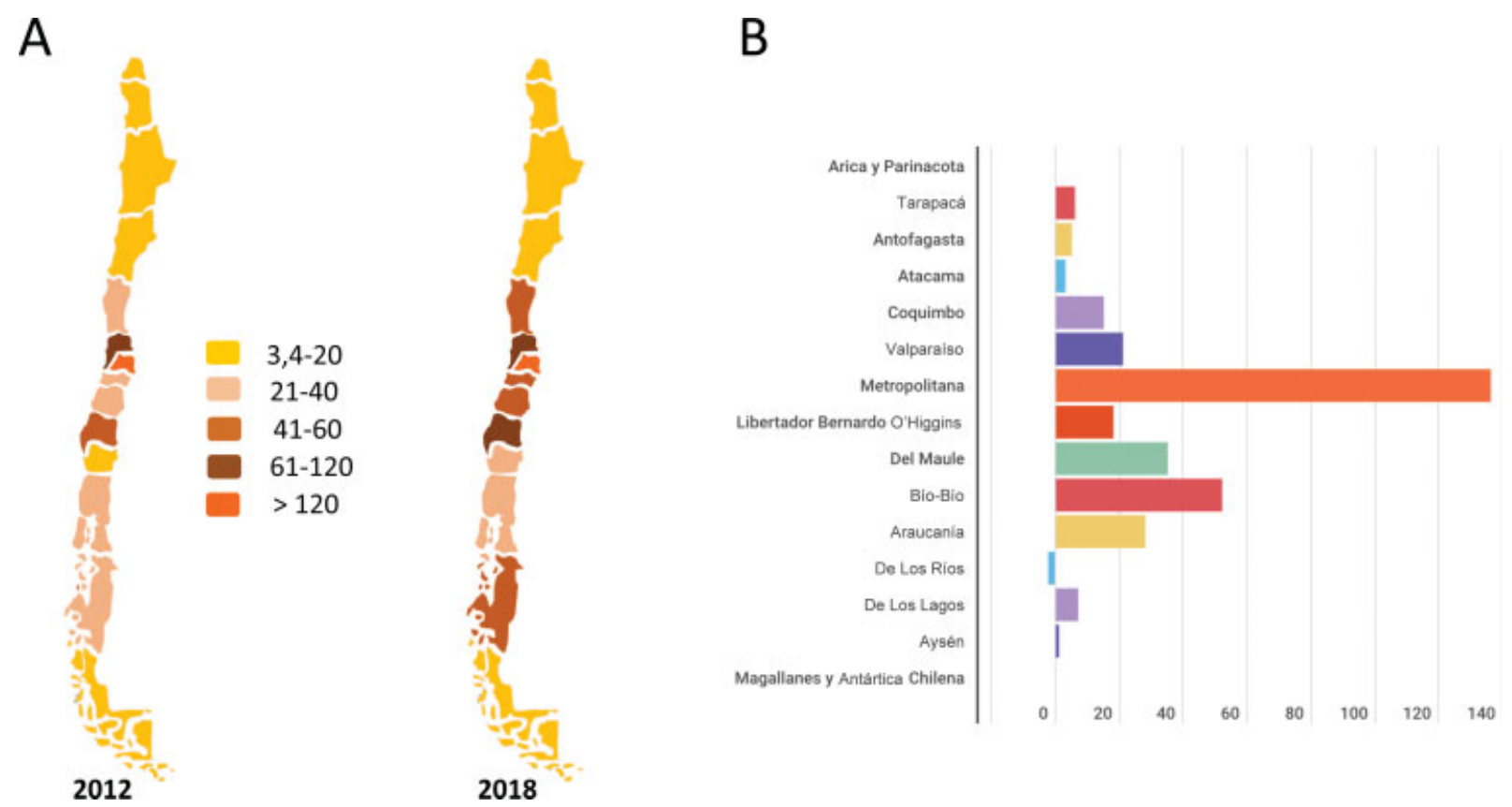

Fig. 1 Osteoartritis (OA) en Chile entre 2012 y 2018. Tasas por 100 mil egresos hospitalarios según datos del Departamento de Estadísticas e Información en Salud (DEIS) del Ministerio de Salud de Chile. (A) Tasas de OA en territorio chileno entre 2012 y 2018. Los colores representan la distribución por tasas. (B) Diferencia de tasas de OA entre 2018 y 2012 en el territorio chileno. El tamaño de la barra representa el aumento en la tasa registrado en 2018 respecto a 2012.

Tabla 3 Porcentaje de osteoartritis según localización anatómica, Chile, 2012-2018

\begin{tabular}{|l|l|l|l|l|l|l|l|}
\hline Osteoartritis & $\mathbf{2 0 1 2}$ & $\mathbf{2 0 1 3}$ & $\mathbf{2 0 1 4}$ & $\mathbf{2 0 1 5}$ & $\mathbf{2 0 1 6}$ & $\mathbf{2 0 1 7}$ & $\mathbf{2 0 1 8}$ \\
\hline Coxartrosis (\%) & 57,1 & 55,0 & 58,7 & 57,2 & 56,0 & 57,1 & 55,0 \\
\hline Gonartrosis (\%) & 28,2 & 31,0 & 28,1 & 31,5 & 31,1 & 32,1 & 35,3 \\
\hline Otras osteoartritis (\%) & 12,8 & 12,3 & 11,5 & 10,1 & 11,6 & 9,6 & 8,6 \\
\hline Poliartrosis (\%) & 1,2 & 1,1 & 1,1 & 0,7 & 0,5 & 0,5 & 0,4 \\
\hline Osteoartritis de la primera articulación carpometacarpiana (\%) & 0,7 & 0,6 & 0,6 & 0,5 & 0,7 & 0,7 & 0,7 \\
\hline
\end{tabular}

Nota: Porcentaje según el total de casos de osteoartritis por año.

afectación son multifactoriales, incluyendo la mayor frecuencia de obesidad, el menor tono muscular, y la mayor laxitud articular. ${ }^{22}$ Además, la prevalencia cambia según la edad, con un gran aumento de los casos después de los 50 años y generándose un plató después de los 70 años. ${ }^{9}$ Aunque no se conoce el motivo de este plató, es posible que las cirugías de reemplazo de articulaciones entre la población de edad avanzada sean más complejas de abordar. $^{20}$
En cuanto a la evolución epidemiológica, un estudio ${ }^{20}$ hecho en Suecia mostró que la OA de cadera fue responsable de la mayor proporción de las hospitalizaciones por OA entre 1998 y 2015. Además, en el Reino Unido la tasa de incidencia en la articulación de la cadera mostró un marcado aumento (3,8\% por año) entre 2000 y 2018. ${ }^{21}$ Estos hallazgos ratifican la importancia epidemiológica de la $\mathrm{OA}$, lo que también queda demostrado en nuestro estudio. 

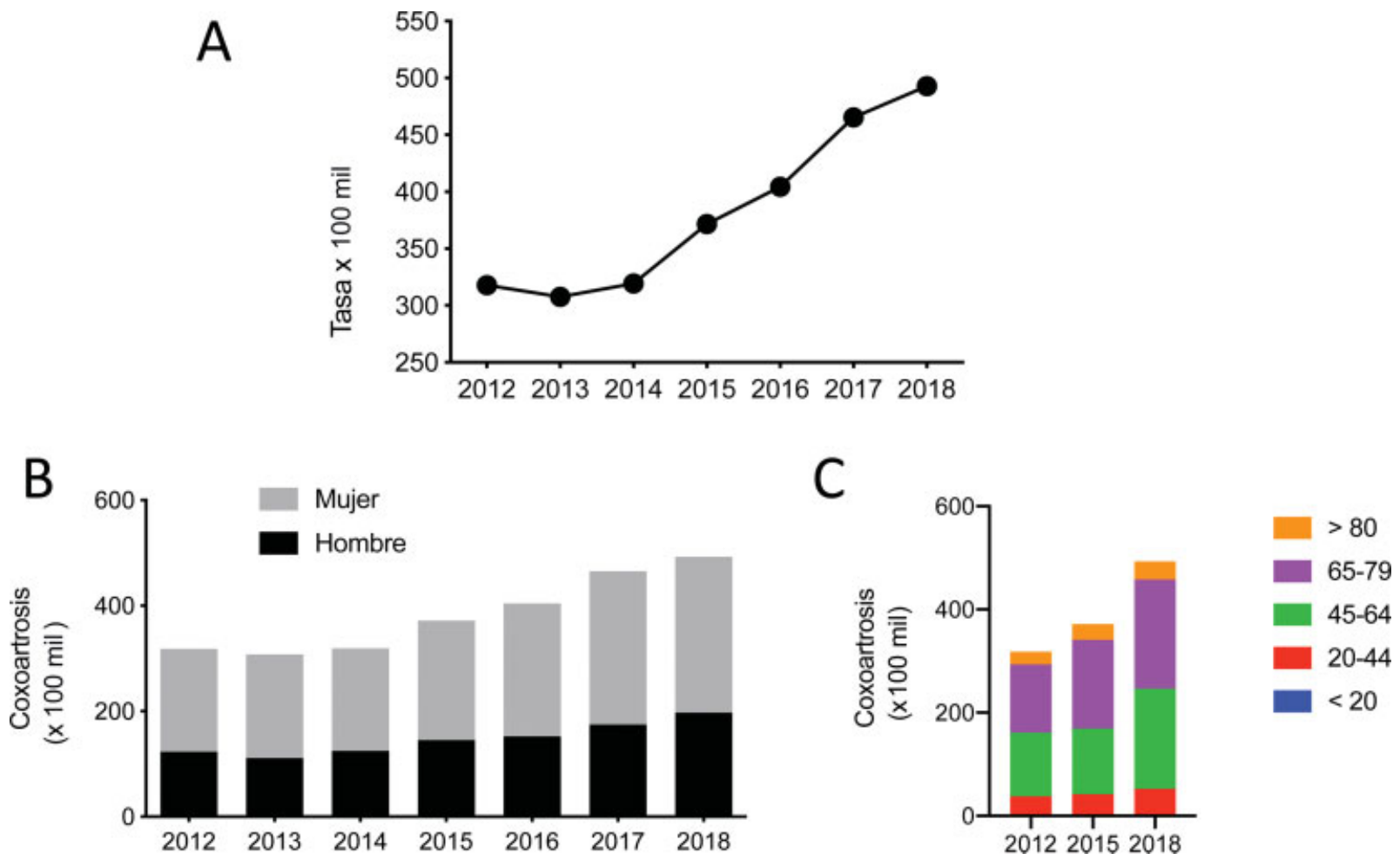

Fig. 2 Osteoartritis de cadera (coxartrosis) en Chile entre 2012 y 2018. Tasas por 100 mil egresos hospitalarios según datos del Departamento de Estadísticas e Información en Salud (DEIS) del Ministerio de Salud de Chile. (A) Evolución de la tasa de coxartrosis. (B) Tasa de coxartrosis según sexo. (C) Tasa de coxartrosis según rango etario en los años 2012, 2015 y 2018. Los colores representan los rangos etarios.
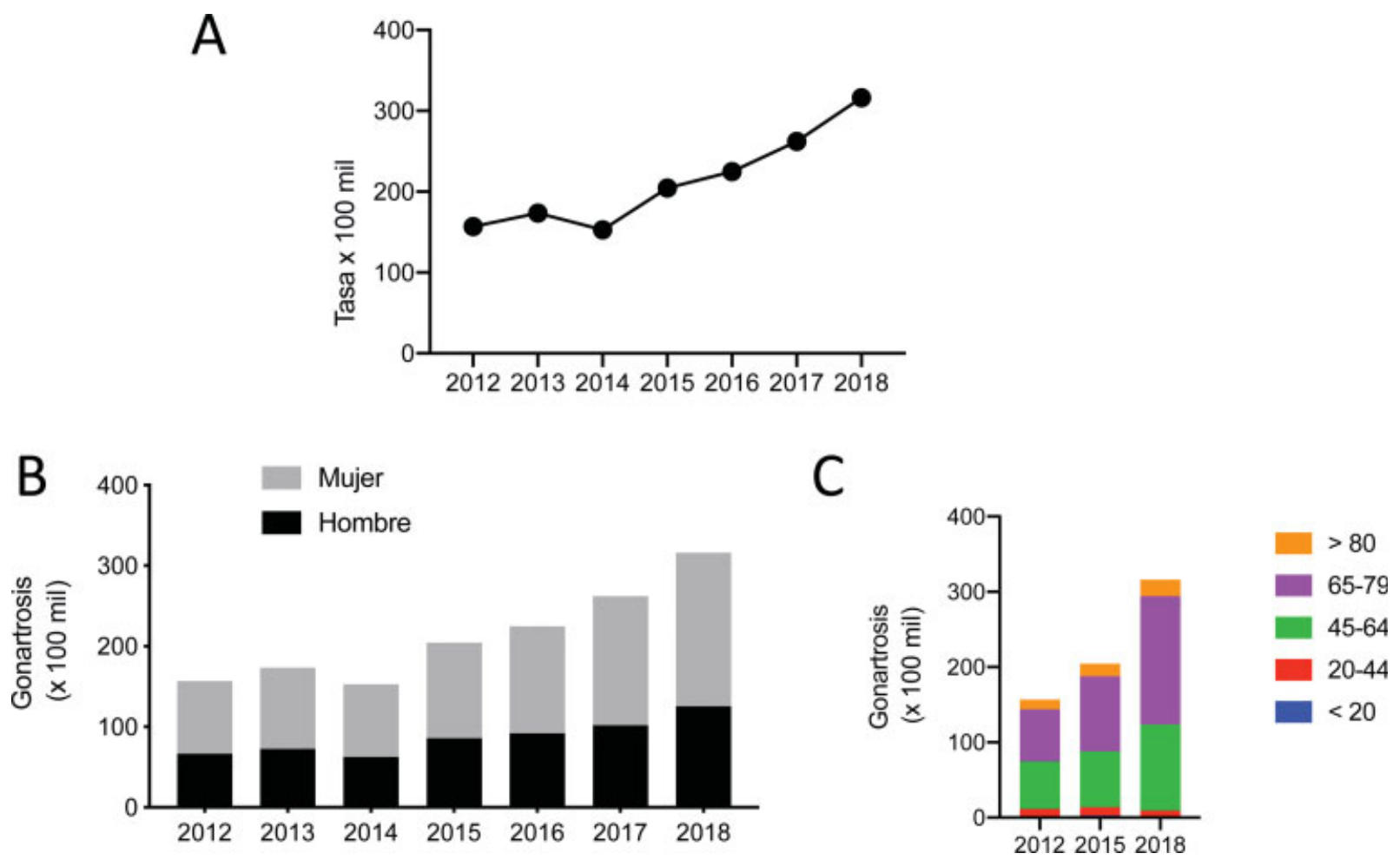

Fig. 3 Osteoartritis de rodilla (gonartrosis) en Chile entre 2012 y 2018. Tasas por 100 mil egresos hospitalarios según datos del Departamento de Estadísticas e Información en Salud (DEIS) del Ministerio de Salud de Chile. (A) Evolución de la tasa de gonartrosis. (B) Tasa de gonartrosis según sexo. (C) Tasa de gonartrosis según rango etario en los años 2012, 2015 y 2018. Los colores representan los rangos etarios. 
A

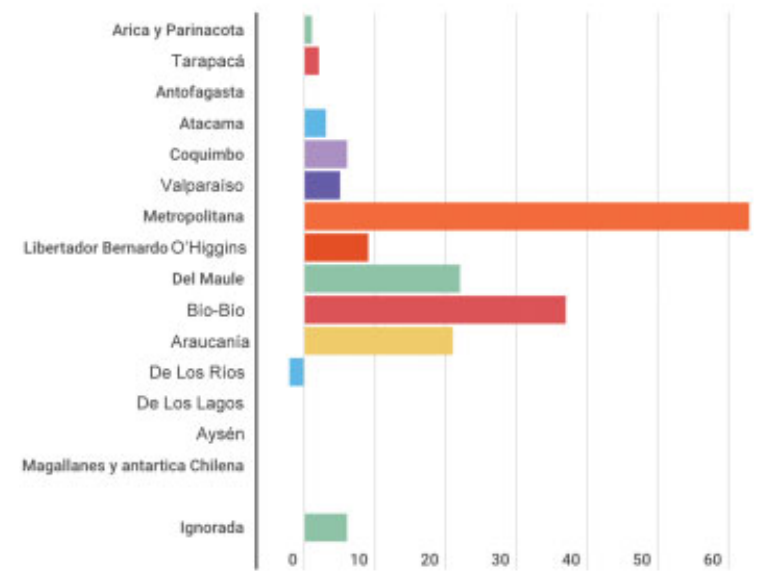

B

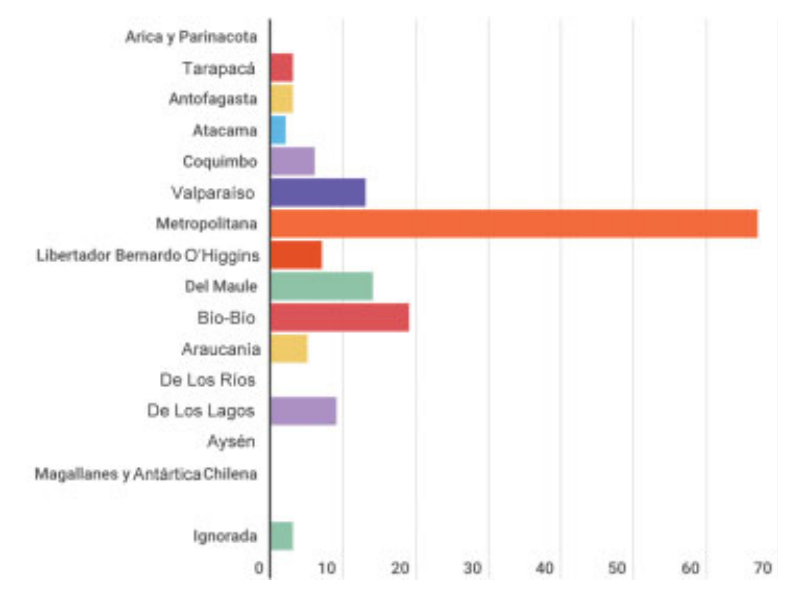

Fig. 4 Osteoartritis de cadera (coxartrosis) y rodilla (gonartrosis) en Chile entre 2012 y 2018. Tasas por 100 mil egresos hospitalarios según datos del Departamento de Estadísticas e Información en Salud (DEIS) del Ministerio de Salud de Chile. (A) Diferencia de tasas de coxoartrosis según región. (B) Diferencia de tasas de gonartrosis según región. El tamaño de la barra representa el aumento en la tasa registrado en 2018 respecto a 2012.

Los resultados arrojan que la mayor cantidad de egresos fue en el sector centro-sur del país. En este sentido, según el censo $2017,{ }^{23} 62,4 \%$ de la población chilena se concentra en las regiones Metropolitana, del Bío-Bío, y Valparaíso, en orden decreciente. Por tanto, las mayores tasas de afectación en estas zonas estarían parcialmente explicadas por la mayor densidad poblacional, así como el mayor acceso a centros hospitalarios de especialidad. En base a esto último, un estudio ${ }^{24}$ de distribución de médicos en el país observó que los médicos se distribuyen de manera similar a la población, sumando las zonas centro y centro-sur del país un $87,7 \%$ de todos los médicos, y la mayor cantidad de traumatólogos (88,93\%). Eso podría explicar la falta de especialistas en regiones más periféricas, lo que obliga a pacientes a trasladar a las zonas centro y centro-sur para sus intervenciones quirúrgicas. Otras posibles causas son la distinta distribución de los factores de riesgo de OA, como la obesidad, la etnia, o el esfuerzo físico, así como también el número de cirujanos, los tiempos de espera quirúrgica, la voluntad de los pacientes, la situación socioeconómica, y las normas culturales..$^{20,24,25}$

En cuanto a la ubicación de la OA, si bien puede afectar cualquier articulación, las más afectadas son la rodilla y la cadera. $^{20,26}$ En ese sentido, se ha reportado que la prevalencia de la OA de cadera en EEUU en 2009 fue del $9,2 \%,{ }^{27}$ en Grecia, del $0,9 \%$ en $2006,{ }^{28}$ y en Croacia, del $23 \%$ en $2000,{ }^{29}$ en adultos mayores de 45 años. Aunque no encontramos reportes individualizados por países de Latinoamérica enfocados a este grupo etario, se mostró que, en 2015 , la OA de cadera afectaba a $1,3 \%$ de adultos mayores (edad promedio: 62,5 años) en un estudio que incluyó a 13 países de Latinoamérica. ${ }^{29}$ Es necesario anotar que las tasas reportadas corresponden a la población total, y no como hemos indicado en este estudio respecto al total de egresos hospitalarios, por lo cual las comparaciones son difíciles de realizar. Sin embargo, es un primer análisis que da una idea clara del comportamiento de la OA de cadera en Chile.

La OA de cadera afecta más al sexo femenino, con predominio en mujeres de más de 45 años, ${ }^{20,21,27}$ debido al efecto protector que tendrían los estrógenos en edades tempranas. ${ }^{26,30}$ Reforzando lo descrito, pero centrada en la edad de la población afectada, una revisión sistemática ${ }^{28} \mathrm{de}$ la prevalencia de OA de cadera según criterios radiográficos informó su aumento gradual con la edad avanzada, más pronunciado después de los 60 años, lo cual confirma evidencias previas en otras poblaciones. ${ }^{20,28}$ Las causas incluyen cambios en el estado funcional de condrocitos, ligamentos, musculatura, y viscoelasticidad articular. ${ }^{30,31}$

Un hallazgo importante de nuestro estudio es la duplicación de la tasa de egresos hospitalarios por OA de cadera en menores de 20 años. A pesar de este aumento, la tasa sigue siendo muy baja. Hubo un crecimiento constante de la carga de morbilidad debida a la OA entre las personas de 15 a 49 años. ${ }^{32}$ Adicionalmente, se ha mostrado aumento de la prevalencia de OA de cadera en menores de 40 años entre 1998 y 2018. ${ }^{20,21,32}$ Es difícil comparar estos estudios previos con nuestros resultados, pero, por lo que sabemos, son los únicos hallazgos publicados que muestran la evolución epidemiológica en individuos jóvenes. A pesar de que no se conocen las posibles causas para este incremento en la población joven, es necesario descartar en primer lugar que se asocie a un error al diagnóstico, ya que el CIE-10 no contempla diagnósticos como sinovitis transitoria de cadera, epifisiolisis de cadera, o enfermedad de Perthes, que son frecuentes en este rango etario. ${ }^{33}$ Además, otras posibles causas serian el pinzamiento femoacetabular (PFA), ${ }^{21}$ o el aumento de las lesiones deportivas, con un consecuente aumento en la indicación quirúrgica en este grupo de pacientes. ${ }^{20}$

Resalta el aumento de las hospitalizaciones por OA de cadera (y rodilla) en la Región de la Araucanía. No conocemos 
las razones para este aumento; sin embargo, dado que $34,3 \%$ de esta población pertenece a pueblos originarios, ${ }^{34}$ es posible sugerir un componente genético. Otro aspecto podría ser la alta prevalencia de obesidad $(56,1 \%)$ y diabetes tipo 2 en la población mapuche, ${ }^{35,36}$ y en particular en las mujeres. En este sentido, es conocido que la obesidad es un factor de riesgo para OA de cadera. ${ }^{20,30}$ Otra posible explicación podría ser el alto estrés biomecánico de la articulación de la cadera al cual es sometida esta población dedicada principalmente al sector silvoagropecuario. ${ }^{25}$

Por otro lado, en cuanto a la OA de rodilla, existen definiciones tanto clínicas como radiográficas; ${ }^{37}$ en estudios internacionales, ${ }^{21,29,38}$ la prevalencia de ha mostrado ser la más alta en comparación con otros tipos de OA. En particular, el estudio de Framingham ${ }^{37}$ mostró que la prevalencia de OA de rodilla fue de $4.9 \%$ en 1987 (en pacientes $\geq 26$ años). En Latinoamérica, no fue posible encontrar datos sobre la prevalencia de OA de rodilla en la población general. Sin embargo, en un estudio ${ }^{29}$ de 2015 que analizó datos de más de 3 mil pacientes en Latinoamérica determinó que la OA de rodilla correspondió al 31.2\% de las OAs evaluadas. A pesar de tener distinta medida, los datos mostrados en este trabajo concuerdan en que la rodilla es una de las principales ubicaciones de OA, siendo responsable en promedio del $31 \%$ de los egresos hospitalarios por OA en Chile.

Asimismo, en el Reino Unido, la OA de rodilla mostró un aumento promedio en la incidencia del $2.9 \%$ anual entre 2000 y $2018 .{ }^{21}$ A diferencia de este último estudio, ${ }^{20}$ nuestro reporte determinó un aumento promedio de $13 \%$ en la tasa de OA de rodilla por egresos hospitalarios en el periodo analizado. Posibles explicaciones para estos hallazgos son las diferencias tanto demográficas como étnicas de la población chilena en comparación con la inglesa. Asimismo, podrían contribuir otras variables no analizadas, como los criterios diagnósticos, y la experiencia quirúrgica del equipo médico. $^{21}$ Por otro lado, estudios previos ${ }^{20,21}$ muestran que las hospitalizaciones por OA de rodilla son más prevalentes en el sexo femenino y en los rangos etarios más altos (65 a 79 años), lo cual se ratifica en nuestro estudio.

Reconocemos que nuestro estudio tiene limitaciones, como el hecho de que se muestran tasas por egresos hospitalarios y no por población, lo que dificulta comparaciones. Igualmente, la base de datos no incluye a pacientes ambulatorios. Además, dado que usamos una base de datos general, y no un análisis caso a caso, no podemos discriminar entre reingresos, recidivas, y nuevos procedimientos en un mismo paciente. Existe un inevitable sesgo de inclusión al usar una base de datos de egresos por hospitalizaciones, ya que la garantía GES sólo incluye a pacientes con $\mathrm{OA}$ de cadera de resolución quirúrgica. Por otro lado, el otro grupo que recibe tratamiento quirúrgico para su patología son los pacientes con OA de rodilla, que, aunque no estén cubiertos por el GES, reciben tratamiento en listas de espera más largas. En este contexto, a pesar de la importancia de la base de datos utilizada, ésta no permite discriminar entre un aumento en el tiempo en la frecuencia de OA o un aumento en una determinada prestación. Tampoco se puede conocer, a partir de esta base de datos pública, el detalle de las distintas articulaciones afectadas por OA que requirieron hospitalización. A pesar de esto, los resultados dan una idea general y coherente sobre la afectación de la población chilena por OA. Este estudio es el único estudio que muestra la distribución de OA tanto por ubicación anatómica como por sexo, edad y ubicación geográfica en el territorio chileno.

En conclusión, entre 2012 y 2018, las hospitalizaciones por $\mathrm{OA}$ en Chile aumentaron en forma sustancial $\mathrm{y}$ progresiva, principalmente las por OAs de cadera y rodilla en el grupo de mujeres de edad avanzada de la zona centro del país. Sin embargo, también llama la atención la duplicación de la tasa en individuos jóvenes. La evidencia en relación a la OA en Chile es escasa; por tanto, es necesario mayor investigación en cuanto a la presentación clínica, la evolución epidemiológica, y el impacto en la sociedad de esta patología prevalente. Nuestros resultados aportan evidencias al conocimiento de esta patología poco estudiada en nuestro país, para el diseño de nuevas medidas de salud pública, y también resalta la necesidad de mejorar el registro de códigos en atención ambulatoria.

\section{Financiación}

CE es apoyado financieramente por Fondecyt Regular 1200250 .

\section{Conflicto de Intereses}

Los autores no presentan conflicto de intereses que declarar.

\section{References}

1 Cillero Pastor B, López Armada M, Caramés B, Blanco García F. Fisiopatología de la artrosis: ¿Cuál es la actualidad? Rev Esp Reumatol 2004;31(06):379-393

2 Martínez Figueroa R, Martínez Figueroa C, Calvo Rodriguez R, Figueroa Poblete D. Osteoartritis (artrosis) de rodilla. Rev. Chil. Ortop. y Traumatol. 2015;56(03):45-51

3 Hunter DJ, McDougall JJ, Keefe FJ. The symptoms of osteoarthritis and the genesis of pain. Rheum Dis Clin North Am 2008;34(03): 623-643

4 Nelson AE. Osteoarthritis year in review 2017: clinical. Osteoarthritis Cartilage 2018;26(03):319-325

5 Garriga XM. Definición, etiopatogenia, clasificación y formas de presentación. Aten Primaria 2014;46(Suppl 1):3-10

6 Moskowitz RW. The burden of osteoarthritis: clinical and qualityof-life issues. Am J Manag Care 2009;15(8, Suppl)S223-S229

7 Kloppenburg M, Berenbaum F. Osteoarthritis year in review 2019: epidemiology and therapy. Osteoarthritis Cartilage 2020;28(03): 242-248

8 Neogi T. The epidemiology and impact of pain in osteoarthritis. Osteoarthritis Cartilage 2013;21(09):1145-1153

9 Neogi T, Zhang Y. Epidemiology of osteoarthritis. Rheum Dis Clin North Am 2013;39(01):1-19

10 Arden N, Nevitt MC. Osteoarthritis: epidemiology. Best Pract Res Clin Rheumatol 2006;20(01):3-25

11 Altman RD. Early management of osteoarthritis. Am J Manag Care 2010;16(Suppl Management):S41-S47

12 Woolf AD, Pfleger B. Burden of major musculoskeletal conditions. Bull World Health Organ 2003;81(09):646-656

13 Danielsson L, Hernborg J. Morbidity and mortality of osteoarthritis of the knee (gonarthrosis) in Malmö, Sweden. Clin Orthop Relat Res 1970;69(69):224-226 
14 Peláez-Ballestas I, Sanin LH, Moreno-Montoya J, et al; Grupo de Estudio Epidemiológico de Enfermedades Músculo Articulares (GEEMA) Epidemiology of the rheumatic diseases in Mexico. A study of 5 regions based on the COPCORD methodology. J Rheumatol Suppl 2011;86:3-8

15 Senna ER, De Barros AL, Silva EO, et al. Prevalence of rheumatic diseases in Brazil: a study using the COPCORD approach. J Rheumatol 2004;31(03):594-597

16 Gamboa DR, et al. Prevalencia de enfermedades reumatologicas y discapacidad en una comunidad urbano-marginal : resultados del primer estudio CoPCord en el Perú. Rev Per Reum. 2009;15(01): 40-46

17 Bilbeny N. Dolor Crónico En Chile. Rev Med Clin Las Condes 2019; 30(06):397-406

18 GOBIERNO DE CHILE. Ministerio de Salud. Decreto 170. Aprueba Garantias Explicitas en Salud del Regimen General de Garantias en Salud. 2005

19 MINISTERIO DE SALUD. Guía Clínica Tratamiento Médico en personas de 55 años y más con artrosis de cadera y/o rodilla, leve o moderada. 2009

20 Kiadaliri AA, Rinaldi G, Lohmander LS, Petersson IF, Englund M. Temporal trend and regional disparity in osteoarthritis hospitalisations in Sweden 1998-2015. Scand J Public Health 2019;47(01):53-60

21 Morgan OJ, Hillstrom HJ, Ellis SJ, et al. Osteoarthritis in England: Incidence Trends From National Health Service Hospital Episode Statistics. ACR Open Rheumatol 2019;1(08):493-498

22 Solis Cartas U, et al. Rasgos demográficos en la osteoartritis de rodilla. Rev. Cuba. Reumatol. RCuR 2015;17(01):32-39

23 Instituto Nacional de Estadísticas. Resultados definitivos Censo 2017(Censo de Población y Vivienda 2017). https://www.censo 2017.cl/wp-content/uploads/2017/12/Presentacion_Resultados_ Definitivos_Censo2017.pdf

24 Guillou M, Carabantes C J, Bustos F V. Disponibilidad de médicos y especialistas en Chile. Rev Med Chil 2011;139(05):559-570

25 Oficina de Estudios y Políticas Agrarias. Region de la Araucanía, Informacion regional 2018. 2018

26 Felson DT. Epidemiology of hip and knee osteoarthritis. Epidemiol Rev 1988;10(01):1-28
27 Lespasio MJ, Sultan AA, Piuzzi NS, et al. Hip Osteoarthritis: A Primer. Perm J 2018;22:17-084

28 Dagenais S, Garbedian S, Wai EK. Systematic review of the prevalence of radiographic primary hip osteoarthritis. Clin Orthop Relat Res 2009;467(03):623-637

29 Reginato AM, Riera H, Vera M, et al; Pan-American League of Associations for Rheumatology (PANLAR) Osteoarthritis Study Group. Osteoarthritis in Latin America: Study of Demographic and Clinical Characteristics in 3040 Patients. J Clin Rheumatol 2015;21(08):391-397

30 Andrianakos AA, Miyakis S, Trontzas P, et al; ESORDIG study group. The burden of the rheumatic diseases in the general adult population of Greece: the ESORDIG study. Rheumatology (Oxford) 2005;44(07):932-938

31 Ingvarsson T. Prevalence and inheritance of hip osteoarthritis in Iceland. Acta Orthop Scand 2001;72(01):1-46

32 Ackerman IN, Kemp JL, Crossley KM, Culvenor AG, Hinman RS. Hip and knee osteoarthritis affects younger people, too. J Orthop Sports Phys Ther 2017;47(02):67-79

33 Hernández BJC. Coxalgia en el niño: Diagnóstico diferencial. Rev Chil Pediatr 2004;75(02):177-180

34 Biblioteca del Congreso Nacional de Chile. Indicadores sociodemográficos y económicos Región de La Araucanía. 2017

35 Pérez-Bravo F, Carrasco E, Santos JL, Calvillán M, Larenas G, Albala C. Prevalence of type 2 diabetes and obesity in rural Mapuche population from Chile. Nutrition 2001;17(03): 236-238

36 Pérez F, Carrasco E, Santos JL, Calvillán M, Albala C. Prevalencia de obesidad, hipertensión arterial y dislipidemia en grupos aborígenes rurales de Chile. Rev Med Chil 1999;127(10): 1169-1175

37 Felson DT, Naimark A, Anderson J, Kazis L, Castelli W, Meenan RF. The prevalence of knee osteoarthritis in the elderly. The Framingham Osteoarthritis Study. Arthritis Rheum 1987;30 (08):914-918

38 Schäfer T, Pritzkuleit R, Jeszenszky C, et al. Trends and geographical variation of primary hip and knee joint replacement in Germany. Osteoarthritis Cartilage 2013;21(02): 279-288 